\title{
Adaptive Droop Control for Effective Power Sharing in Multi-terminal DC (MTDC) Grids
}

\author{
Nilanjan Ray Chaudhuri, Member, IEEE, and Balarko Chaudhuri, Senior Member, IEEE
}

\begin{abstract}
Following a converter outage in an MTDC grid, it is critical that the healthy converter stations share the power mismatch/burden in a desirable way. A fixed value of powervoltage droop in the DC link voltage control loops can ensure proper distribution according to the converter ratings. Here a scheme for adapting the droop coefficients to share the burden according to the available headroom of each converter station is proposed. Advantage of this adaptive (variable) droop scheme for autonomous power sharing is established through transient simulations on an MTDC grid with four bipolar converters and DC cable network with metallic return. Results for both rectifier and inverter outages under two different scenarios are presented. Post contingency steady-state operating points obtained from transient simulation are shown to be consistent with those derived analytically. Impact of varying droop coefficients on the stability of the MTDC grid is established. An averaged model in Matlab/SIMULINK which has been validated against detailed switched model in EMTDC/PSCAD is used for the stability and modal analysis.
\end{abstract}

Index Terms-Multi-terminal DC (MTDC), Autonomous power sharing, Droop, Voltage source converter (VSC), Stability, Modal analysis

\section{INTRODUCTION}

$\mathbf{M}$ ULTI-TERMINAL DC (MTDC) grids could form the backbone of a pan-European interconnected system in future. A sub-sea MTDC grid is planned around the North sea to tap the rich wind resource of the region and also interconnect the UK and Nordic pool with continental Europe [1], [2]. Apart from the huge investment and key technological barriers like lack of commercial availability of DC circuit breakers for high power levels, system operators are concerned about the unknown impact of MTDC grid operation and control on the onshore AC systems.

One particular issue is how would an MTDC grid react to loss/ outage of one or more converter stations and the resulting power imbalance. Sharing the burden of such a loss/power mismatch has to be appropriate in order to minimize the impact on the neighboring AC system. The concept of droop control [3], [4], [5] has been proposed for power sharing and frequency support [6] through MTDC grids. Fixed values of droop constants, primarily based on the individual rating of the converters have been used. However, this does not consider the actual loading condition and hence the headroom (difference between the rated capacity and actual loading) available to share the additional burden.

In this paper, we propose an adaptive (variable) droop control scheme for appropriate power sharing taking into account

\footnotetext{
N.R. Chaudhuri and B. Chaudhuri are with Imperial College London, London SW7 2BT, UK (e-mail:
} n.chaudhuri@imperial.ac.uk, b.chaudhuri@imperial.ac.uk). the available headroom and thus avoid possible overloading. The converters which are already operating close to the limits would not try to share the burden of the loss to the extent of the other converters with higher spare capacity or headroom.

Similar to converter-fed AC networks (e.g. a microgrid) [7], droop control impacts the stability of MTDC grids. However, such impact of overall stability of the MTDC grid has not been analyzed in any of the earlier work except through simulation studies. For a fixed droop scheme, thorough stability analysis might not be essential as long as stability is ensured for the chosen fixed values through prior simulation. But for adaptive droop, the stability region needs to be ascertained through modal analysis. In this work, we have used the averaged model in Matlab/SIMULINK to perform the stability and modal analysis. Validation of this averaged model against detailed switched model in EMTDC/PSCAD provided the required confidence in this analysis.

To illustrate the benefits of the proposed adaptive droop control scheme, a case study is performed with an MTDC grid having four bipole converter stations and DC cable network with metallic return. Comparison between fixed and adaptive droop has been made in the backdrop of no power sharing control (i.e. without droop control) through transient simulation in EMTDC/PSCAD. Out of several scenarios considered, a set of simulation results for both rectifier and inverter outages are presented under two different operating scenarios. Postcontingency steady-state operating points are derived analytically for both fixed and variable droop schemes. Analytical results are shown to be consistent with those observed in transient simulation. Impact of droop constant values on the stability of the MTDC grid is demonstrated with a view to guarantee the stability zone.

\section{Modeling}

\section{A. Converter Modeling}

We have followed a similar approach as in [8] for modeling the converters which is described here briefly. Sinusoidal PWM was considered as the switching strategy and the converters were represented by their averaged model [9] in Matlab/SIMULINK.

The model of positive/negative pole of the $j^{\text {th }}$ converter station is shown in Fig. 1(a) in a synchronously rotating reference frame $d^{\prime}-q^{\prime}$. The $d^{\prime}$-axis is locked with the voltage $E_{a c j}$ on the AC system side of the converters (Fig. 1(b)) to ensure decoupled control of the active and reactive power. All notations in the modified reference frame are henceforth denoted with a prime. 


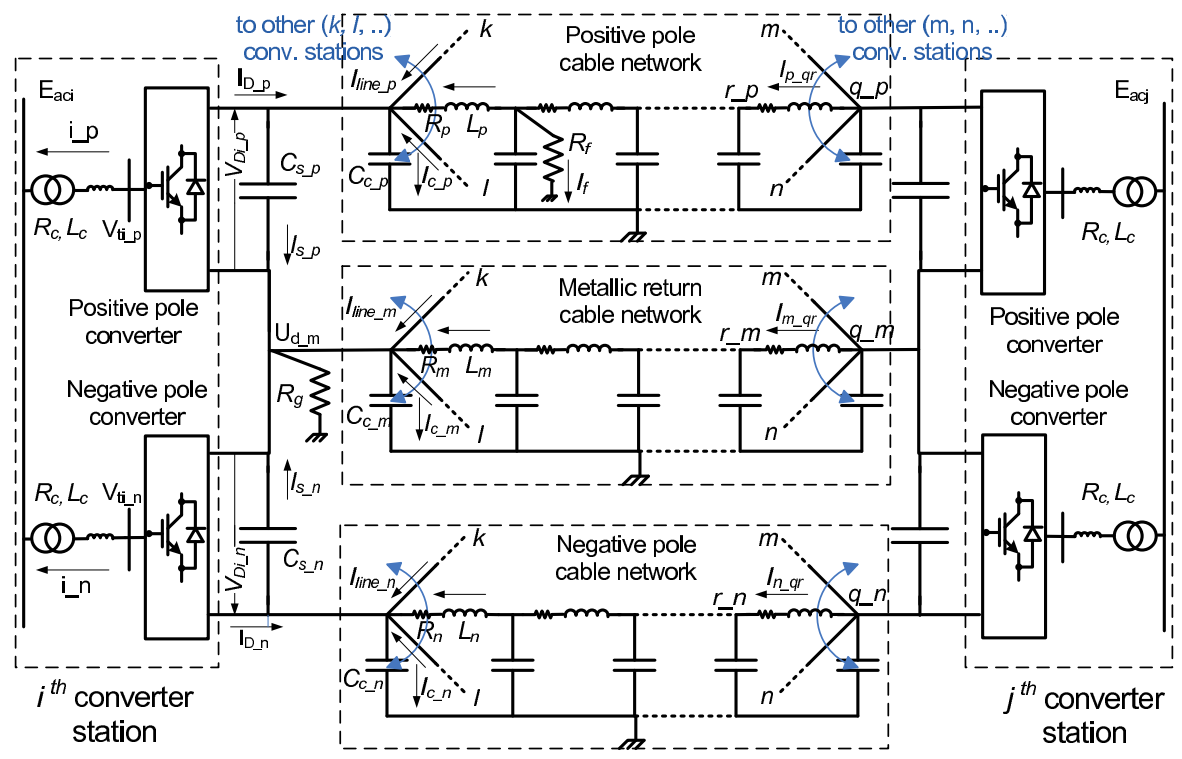

Fig. 2. DC cable network modeling with cascaded pi sections

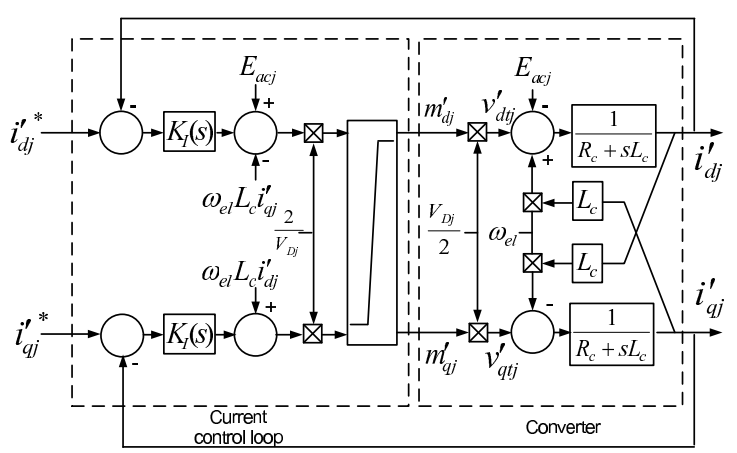

(a)

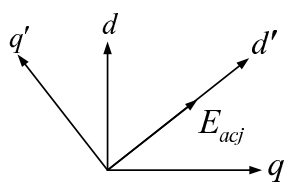

(b)

Fig. 1. (a) VSC MTDC converter model with overall control structure for the $j^{t h}$ converter (b) modified reference frame for decoupled control

The AC system connected to the MTDC grid is modeled in $d-q$ reference frame as shown in Fig. 1(b) which needs to be transformed to $d^{\prime}-q^{\prime}$ frame and back while interfacing the MTDC grid variables with those of the AC system. From Fig. 1 the dynamics of the AC side of the converters in $d^{\prime}-q^{\prime}$ frame can be written as:

$$
\begin{array}{r}
v_{d t j}^{\prime}-R_{c} i_{d j}^{\prime}-L_{c} \frac{d i_{d j}^{\prime}}{d t}+L_{c} \omega_{e l} i_{q j}^{\prime}=E_{a c j} \\
v_{q t j}^{\prime}-R_{c} i_{q j}^{\prime}-L_{c} \frac{d i_{q j}^{\prime}}{d t}-L_{c} \omega_{e l} i_{d j}^{\prime}=0
\end{array}
$$

where, $v_{d t j}^{\prime}=m_{d j}^{\prime} \frac{V_{D j}}{2}, v_{q t j}^{\prime}=m_{q j}^{\prime} \frac{V_{D j}}{2}$ and $R_{c}, L_{c}$ are the aggregated resistance and inductance of the converter transformer and phase reactors. Note that, $m_{d j}^{\prime}$ and $m_{q j}^{\prime}$ are the modulation indices for converter control [10].

Decoupled current control strategy [8] as shown in Fig. 1(a) is used for the converters where following relations hold:

$$
P_{j}^{*}=\frac{3}{2} E_{a c j} i_{d j}^{\prime *}, Q_{j}^{*}=-\frac{3}{2} E_{a c j} i_{q j}^{\prime *}
$$

\section{B. DC Cable Network Modeling}

An asymmetric bipole MTDC grid with positive, negative and metallic return network was considered which is generic enough to handle different grounding mechanisms and 'unbalance on the DC side' as a result of outage of one or more poles and/ or converters. A cascaded pi section approximation of the distributed model of the cable as shown in Fig. 2 was used and the minimum number of such sections required (four in this case) was ascertained based on the compromise between accuracy and the cost of computational burden.

Fig. 2 shows the interconnection between the $i^{\text {th }}$ and the $j^{\text {th }}$ converter stations through the positive, negative and the metallic return cable. Each of the converter poles can be connected to the corresponding poles of any number of converter stations.

The mathematical model of the cable network can be described by the set of differential and algebraic equations (DAEs) shown in a block diagram in Fig. 3. The incidence matrix $C C I_{P}$ relates the vector of $R-L$ branch currents flowing from any general node $q \_p$ to $r \_p$ (i.e. $\bar{I}_{p_{-} q r}$ ) with the total node current injection vector $\bar{I}_{\text {line_p }}$ (see, Fig. 2 ) by the relation

$$
\bar{I}_{\text {line_p }}=C C I_{P} \times \bar{I}_{p_{\_} q r}
$$

where, $C C I_{P}(i, j)=1(-1)$, if the $i^{\text {th }}$ cable enters (leaves) the $j^{\text {th }}$ node and $C C I_{P}(i, j)=0$, otherwise. Note that $C C I_{P}=$ $-C C U_{P}^{T}$.

The governing DAEs for the MTDC grid can be written from Figs 2 and 3 and are not repeated here since modeling is not the focus of this paper. The readers are referred to [11], [12] for further details. It should be noted that the converter outage was simulated by opening both the DC side and the $\mathrm{AC}$ side breakers at the respective converter pole. However, 


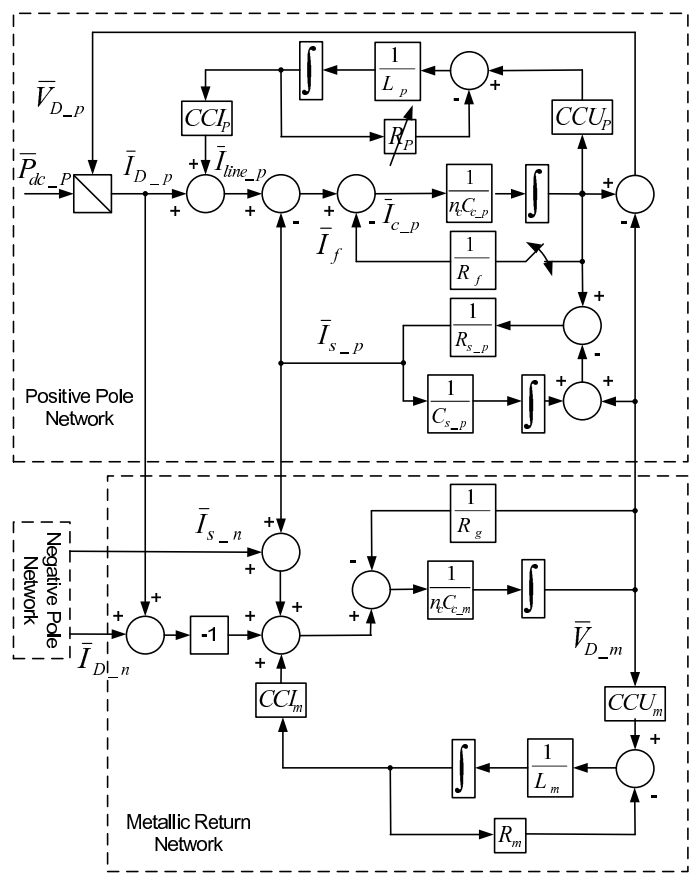

Fig. 3. Block diagram of the DC cable network dynamic model

the DC cable connections to the switchyard of the affected converter were retained.

\section{Autonomous Power SHARING IN MTDC GRIDS}

\section{A. Fixed Droop}

For point-to-point VSC-HVDC links, the usual practice is to operate one of the converter stations in active power control mode to ensure the scheduled power exchange. The DC link voltage is maintained by the other station which consequently supplies the resistive losses in the DC link, thus acting as a slack/swing converter station.

For an MTDC grid with more than two converter stations it is desirable that following an outage of one or more converters, all the remaining ones should share the resulting power imbalance in certain appropriate proportion [3], [4], [5]. Thus all the converter stations should operate in DC link voltage control mode (or as swing converter) rather than trying to follow their respective active power references. However, set values of DC link voltage references at all the converter stations could be conflicting unless they are modified properly depending on the reference and actual power and DC link voltage.

The situation is similar to parallel operation of generators in an AC system where power-frequency droop is used in the governor control loop to avoid potential conflict in frequencies. Along the same lines, active power-DC link voltage droop can be used to modify the reference values of DC link voltages $V_{D j}^{2}$ at each converter station (under voltage control mode) as shown in Fig. 4. This is consistent with the development first described in [5].

Using the modified voltage reference a PI controller derives the $d$-axis current reference $I_{d j}$ for the current control loop of the converters. Squared values of half of the the reference

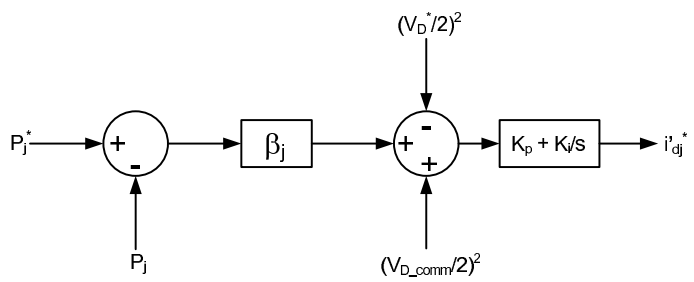

Fig. 4. Active power-DC link voltage droop for sharing of power imbalance in the $j^{\text {th }}$ converter station

and measured DC link voltages $\left(\frac{V_{D j}{ }^{*}}{2}\right)^{2},\left(\frac{V_{D-\text { comm }}}{2}\right)^{2}$ are normally used for the voltage control loops [13]. Also local voltage feedback results in a non-unique power flow solution for the MTDC grid. Hence, a common voltage $V_{D-c o m m}$ (at any converter station) is communicated as feedback signal to the control loop of the remaining converter stations as was first introduced in [5].

The values of the droop constants $\beta_{j}$ at different converter stations determine how a power imbalance is shared among the converters. Identical values of $\beta_{j}$ would result in equal sharing among the converters. For unequal $\beta_{j}$, the ones with higher values would have dominant contribution from active power $\left(P_{j}{ }^{*}\right)$ control loop. Smaller $\beta_{j}$ would ensure lesser deviation in DC link voltages, but it might adversely impact the stability as discussed later in Section VI.

Although a fixed or predefined values of the droop constants $\beta_{j}$ can be chosen according to the ratings of the individual converter station [3], [4], [5], it does not consider the actual loading condition and hence the headroom available for sharing the additional burden arising due to a power imbalance situation. This problem with fixed droop can be avoided using an adaptive droop scheme described in the next subsection.

\section{B. Adaptive (variable) Droop}

For a fixed droop scheme, shown in Fig. 4, the droop coefficients are usually dependent only on the respective converter ratings. Therefore, for an MTDC grid with equally rated converter stations the sharing of burden following a converter outage will be equally distributed as elaborated in Section IV. However, under a particular operating condition, all the converter stations might not be equally loaded and hence some of them may not be able to participate in power sharing equitably. Therefore, it is desirable that the available converters should participate in power sharing depending on the headroom (difference between the rated capacity and present loading) available in addition to their ratings. To address this issue the contribution from the power control loop in Fig. 4 is modified as shown in Fig. 5. It is proposed that the droop coefficient be calculated based on a function of the normalized available headroom of each converter defined as:

$$
\beta_{j}=\beta_{j}^{0}\left(\frac{\Re_{\text {base }}}{\mathrm{H}_{j}}\right)^{\lambda}
$$

where, $\mathrm{H}_{j}=\Re_{j}-\left|P_{j}\right|$, is the available headroom of the $j^{\text {th }}$ converter of rating $\Re_{j}, \beta_{j}^{0}$ is the nominal droop which is in inverse proportion to the converter ratings, $\Re_{\text {base }}$ is the maximum value of all converter ratings and $\lambda$ is a user 


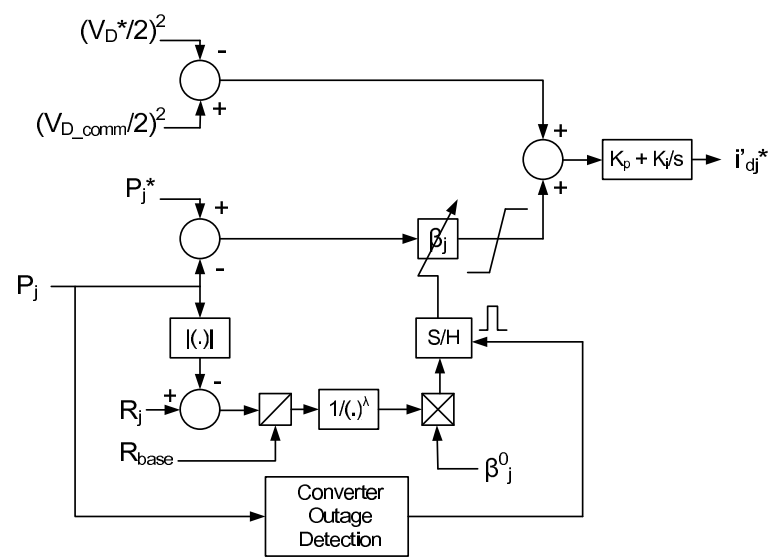

Fig. 5. Block diagram of the proposed variable droop scheme in the $j^{\text {th }}$ converter station

defined positive constant. The above function is inverse nonlinear in $P_{j}$ and results in higher droop values for lower available headroom. This ensures that converters which are already operating very close to the operational limit would not try to share the burden of a lost converter (or due to loss of a cable) to the extent shared by the converters with higher spare capacity or headroom.

It is also anticipated that if the MTDC grid enters into power market in future, the converter station owners may want to use this spare capacity as an ancillary service and share the power mismatch appropriately. Therefore, due to both physical and market related limitations, variable droop constant will be an important aspect to have within HVDC control system intended for future MTDC grids.

The sequence of events followed in the proposed approach are stated below:

1) Use the droop coefficients $\beta_{j}^{(k)}$ calculated based on the pre-contingency steady state of the $k^{t h}$ converter outage (outage in the immediate past).

2) Keep calculating the normalized headroom dependent droop coefficient $\beta_{j}^{(k+1)}$ online but do not use them until the next $(k+1)^{t h}$ outage.

3) Upon detection of a converter outage, command the sample and hold function to invoke the droop values $\beta_{j}^{(k+1)}$ calculated just before the $(k+1)^{t h}$ event.

\section{Post-CONTINGEnCY Operation}

In this section an analytical derivation of the postcontingency operating point in an MTDC network with $n$ converter stations is presented. The analysis is applicable to both the positive and negative pole converters and is broadly similar to [5] except for the variable droop considerations.

Let us assume $P_{j}$ is the real power at the PCC of the $j^{\text {th }}$ converter and $P_{j}^{*}$ is the corresponding reference power. The reference setting of the converters are chosen such that $\sum_{j} P_{j}^{*}=0$. Moreover, the total real power loss in the DC cable network $P_{\text {loss }}=-\sum_{j} P_{j}$. It can be seen from Fig. 4 that under steady state,

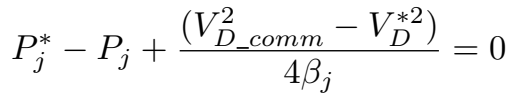

$$
\sum_{j}\left(P_{j}^{*}-P_{j}\right)+\frac{\left(V_{D \_c o m m}^{2}-V_{D}^{* 2}\right)}{4} \sum_{j} \frac{1}{\beta_{j}}=0
$$

From (6) and (7) we get,

$$
\begin{gathered}
V_{D_{-} \text {comm }}^{2}-V_{D}^{* 2}=-4 \frac{P_{\text {loss }}}{\sum_{j} \frac{1}{\beta_{j}}} \\
P_{j}=P_{j}^{*}+\frac{-P_{\text {loss }}}{\beta_{j} \sum_{j} \frac{1}{\beta_{j}}}
\end{gathered}
$$

For a fixed droop scheme it is usual to choose $\beta_{j} \Re_{j}=$ $\beta_{i} \Re_{i}, \forall i, j$ where $\Re_{j}$ is the rating of the $j^{\text {th }}$ converter. Modifying (9) we get:

$$
P_{j}=P_{j}^{*}+\frac{-P_{\text {loss }}}{\frac{1}{\Re_{j}} \sum_{j} \Re_{j}}
$$

It is clear from (10) that in case of equal droop coefficients (i.e. equal converter ratings) the converter real powers deviate from its reference equally by the amount $\frac{P_{l o s s}}{n}$.

Following the outage of the $n^{\text {th }}$ converter:

$$
\sum_{j}^{n-1} P_{j}^{*}=-P_{n}^{*}, \sum_{j}^{n-1} P_{j}=-P_{\text {loss }}^{\prime}
$$

where, $P_{\text {loss }}^{\prime}$ is the total DC transmission loss in the postoutage condition. The post-contingency steady state operating point gets modified to:

$$
\begin{gathered}
V_{D_{-} \text {comm }}^{\prime 2}-V_{D}^{* 2}=-4 \frac{\left(P_{\text {loss }}^{\prime}-P_{n}^{*}\right)}{\sum_{j}^{n-1} \frac{1}{\beta_{j}}} \\
P_{j}^{\prime}=P_{j}^{*}+\frac{\left(-P_{\text {loss }}^{\prime}+P_{n}^{*}\right)}{\frac{1}{\Re_{j}} \sum_{j}^{n-1} \Re_{j}}
\end{gathered}
$$

Note that for converters with equal ratings the difference between the pre-outage and the post-outage power for the $j^{t h}$ converter is:

$$
\Delta P_{j}=P_{j}^{\prime}-P_{j}=\left[\frac{P_{\text {loss }}}{n}-\frac{P_{\text {loss }}^{\prime}}{(n-1)}\right]+\frac{P_{n}^{*}}{(n-1)}
$$

Neglecting the bracketted terms, we can say that the remaining $n-1$ converters will share the burden of the $n^{t h}$ converters equally amongst themselves.

For the variable droop scheme mentioned in Section III-B we also take the available headroom $\left(\mathrm{H}_{j}\right)$ of the $j^{\text {th }}$ converter into consideration. Note that, $\mathrm{H}_{j}=\Re_{j}-\left|P_{j}\right|$, not $\mathrm{H}_{j}=$ $\Re_{j}-\left|P_{j}^{\prime}\right|$ since it depends on the pre-contingency operating point as mentioned in Section III-B. After some algebraic manipulations and neglecting the loss terms as in (14) it can be shown that the difference between the pre-outage and the post-outage power for the $j^{t h}$ converter is:

$$
\Delta P_{j} \approx \frac{P_{n}^{*}}{\left(\frac{1}{\Re_{j}}\right)\left(\frac{\Re_{\text {base }}}{\mathrm{H}_{j}}\right)^{\lambda} \sum_{j}^{n-1} \Re_{j}\left(\frac{\mathrm{H}_{j}}{\Re_{\text {base }}}\right)^{\lambda}}
$$

From the above derivations it can be concluded that the adaptive droop will ensure sharing of power mismatch 
amongst the converters based on their respective available headroom. It is to be noted that when all converter ratings are equal, i.e. $\Re_{j}=\Re_{\text {base }}, \forall j$, putting $\lambda=0$ in (15) results in (14) (neglecting loss terms) which corresponds to the fixed droop situation. Post-contingency steady-state operating points observed in the transient simulations (in Section VII) are shown to correspond with (14) and (15) for fixed and variable droop, respectively.

\section{STUdy NETWORK}

The study network consisting of four bipole converter stations connected with $500 \mathrm{~km}$ cables is shown in Fig. 6 . Converter stations \#2 and \#3 are considered to be acting as

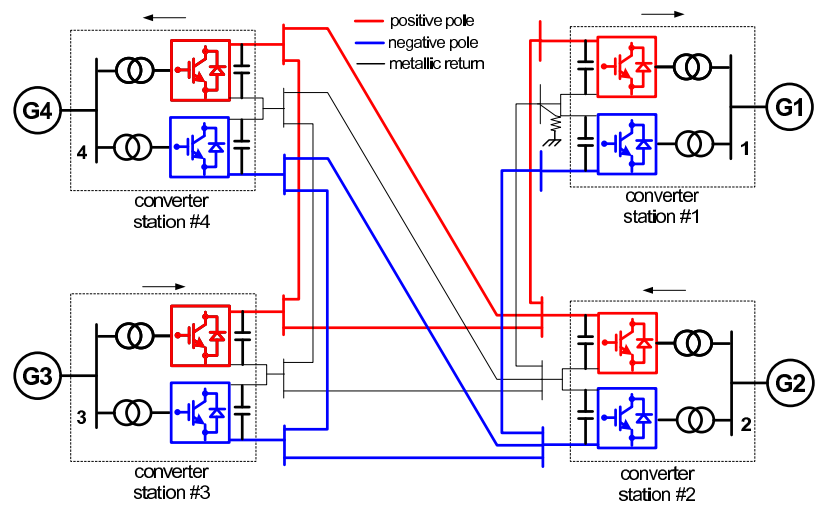

Fig. 6. Bipole MTDC grid with four converter stations

rectifiers while \#1 and \#4 as inverters under nominal condition. For the studies without droop control, station \#1 operates in $V_{d c}-Q$ control mode and keeps the DC link voltage at \pm 350 $\mathrm{kV}$ with unity power factor at the PCC while the rest operate in P-Q control mode. The DC link voltage of station \#1 was chosen as the common reference for the droop scheme. Real power injected into the AC system is considered to be positive and vice versa for the rest of the paper. The metallic return is grounded with a $0.5 \Omega$ resistance at the converter station \#1 while the other stations are left ungrounded. The surrounding $\mathrm{AC}$ system was modeled by ideal $400 \mathrm{kV}$ voltage sources. The steady state operating condition was determined by solving the AC-DC load-flow equations [14].

TABLE I

MTDC GRID PARAMETERS FROM FIGS 1, 2

\begin{tabular}{|c|c||c|c|}
\hline Parameters & Values & Parameters & Values \\
\hline \hline$R_{c}$ & $0.07 \Omega$ & $C_{s_{-} p} / C_{s_{-} n}$ & $0.4 \mathrm{mF}$ \\
\hline$L_{c}$ & $40.0 \mathrm{mH}$ & $R_{s_{-}} / R_{s_{-} n}$ & $1.0 \mu \Omega$ \\
\hline$R_{g}$ & $0.5 \Omega$ & $R_{p} / R_{n} / R_{m}$ & $1.5 \Omega$ \\
\hline$L_{m}$ & $0.30 \mathrm{H}$ & $L_{p} / L_{n}$ & $0.30 \mathrm{H}$ \\
\hline$C_{c_{-} m}$ & $40.0 \mu \mathrm{F}$ & $C_{c_{-} p} / C_{c_{-} n}$ & $14.00 \mu \mathrm{F}$ \\
\hline$R_{f}$ & $0.01 \Omega$ & $K_{I}(s)$ & $300\left(\frac{R_{c}+s L_{c}}{s}\right)$ \\
\hline
\end{tabular}

The parameters of the above study network are shown in the TableI. The AC side parameters $\left(R_{c}, L_{c}\right)$ were chosen from the standard filter reactor data in p.u. The cable parameters were obtained from a $\pm 350 \mathrm{kV}, 500 \mathrm{~km}$ cable model in PSCAD. The controller $K_{I}$ was designed to achieve a closed loop bandwidth of $300 \mathrm{rad} / \mathrm{s}$ since the tracking parameters $\left(i_{d j}^{\prime}\right.$ and $\left.i_{q j}^{\prime}\right)$ are $\mathrm{dc}$ in nature.

\section{Modal Analysis}

The MTDC grid model described by differential-algebraic equations (DAEs) was linearized around a nominal condition and expressed in state-space form as:

$$
\Delta \dot{x}=A \Delta x
$$

where, $\Delta x$ and $A$ are the state-vector and state-matrix, respectively. The right $\left(\phi_{i}\right)$ and left $\left(\psi_{i}\right)$ eigen vectors corresponding to the eigenvalues $\lambda_{i}, i=1,2, \ldots, n$ satisfy:

$$
A \phi_{i}=\lambda_{i} \phi_{i}, \psi_{i} A=\lambda_{i} \psi_{i}
$$

The $k^{\text {th }}$ element of the right eigenvector $\phi_{i}$ measures the activity of the state variable $x_{k}$ in the $i^{t h}$ mode while that of the left eigenvector $\psi_{i}$ weighs the contribution of this activity to the $i^{t h}$ mode [15]. The LAPACK routine in MATLAB were used to compute these.

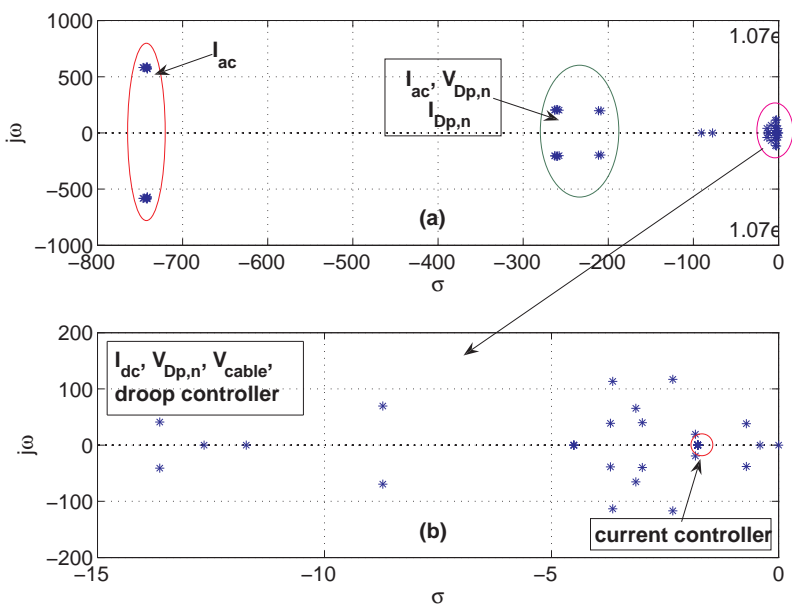

Fig. 7. Clustering of system poles based on participation factors.
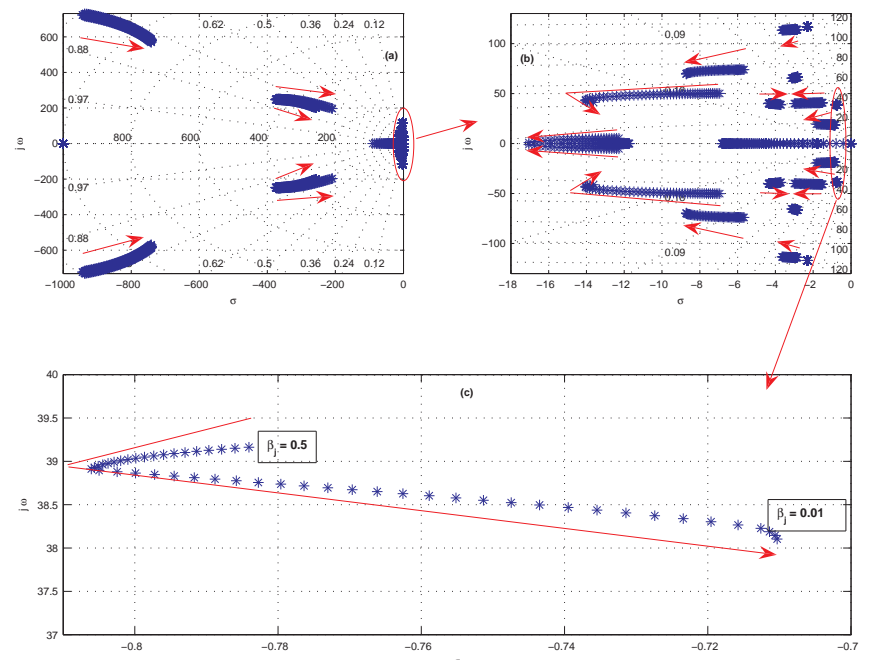

Fig. 8. Root locus of the system with change of droop constants $\left(\beta_{j}\right)$ in the operating condition with the outage of the negative pole converter at converter station \#2.

To get around the scaling problem often the above measures are combined to form the participation matrix, $P$ whose 


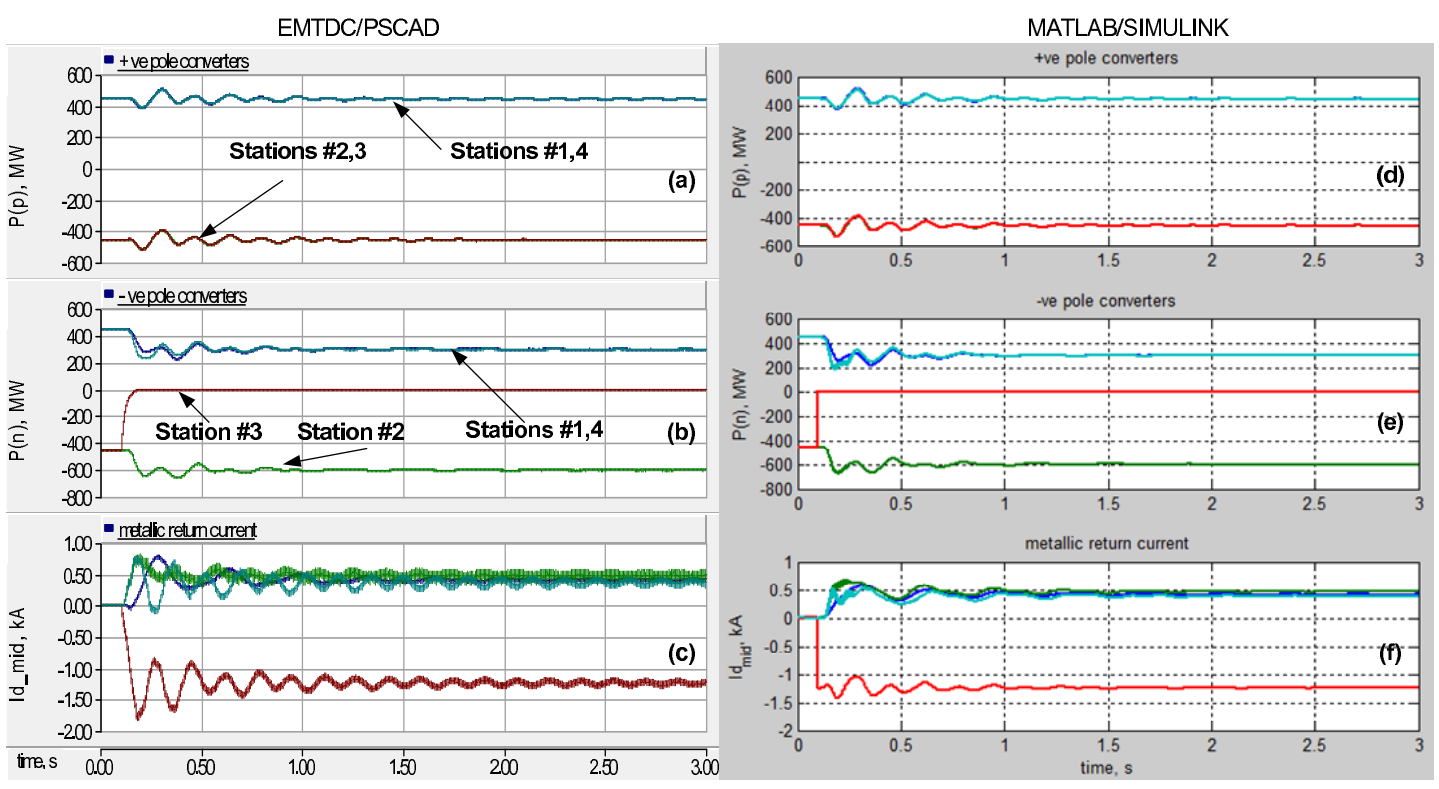

Fig. 9. Validation of the averaged model in MATLAB/SIMULINK with the detailed switched model in EMTDC/PSCAD for autonomous power sharing following the outage of the negative pole converter of the converter station \#3.

elements $p_{k i}=\phi_{k i} \psi_{i k}$ are termed as the participation factor. It is a measure of the relative participation of the $k^{\text {th }}$ state variable in the $i^{\text {th }}$ mode and vice versa [15]. Note that $p_{k i}$ is dimensionless and $\sum_{i=1}^{n} p_{k i}=\sum_{k=1}^{n} p_{k i}=1$.

Participation factor analysis was done to figure out the nature and the root cause of the dynamic response of the MTDC grid. The linearization was done around an operating condition where the outage of the negative pole converter of station \#2 with equal droop constants for all converter stations were considered. It can be seen from the pole map of Fig. 7(a) that the fastest modes are associated with the AC network (modeled by transformer and phase reactor impedance) states and the participation of the DC network and the converter controller states increase as we move towards right on the splane. The lower subplot shows a zoomed view of the critical poles. Most of these are associated with the DC network and the droop controller states except one pole pair which has high participation from the current controllers of the converters.

Fig. 8 shows the locus of the system poles when $\beta_{j}$ of all converters are kept equal and varied from $50 \%$ to $1 \%$. The red arrows indicate the movement of poles due to such variation. It can be seen that the pole clusters having higher participation from the $\mathrm{AC}$ side current move towards right when $\beta_{j}$ is reduced. However, the pole clusters with higher participation from the DC network and droop controller states show a mixed movement (Fig. 7(b)).

The movement of the most critical pole is zoomed in Fig. 7(c). As observed, this pole pair moves towards the left half of the s-plane when $\beta_{j}$ is decreased from 0.5. However, there is a point of inflexion beyond which further decrement results in movement towards the right half of s-plane. A low value of $\beta_{j}$ is desirable since it reduces the steady state deviation in the DC link voltage and stops it from violating the limits at the output of the droop controller as shown in Fig. 5. On the other hand, in the proposed adaptive (variable) droop scheme a lower (higher) headroom might result in higher (lower) droop values. To avoid instability due to large/ small droop values the stability analysis as above is important. Only one typical operating condition is presented here to indicate the need of upper and lower bounds on the droop constants.

\section{Simulation AND Discussion}

\section{A. Validation with Detailed Switched Model}

The averaged model in MATLAB/SIMULINK was validated against a detailed switched model in EMTDC/PSCAD following a converter outage. Fig. 9 shows the power sharing amongst the converter stations following the outage of the negative pole converter in station \#3. A fixed droop scheme (shown in Fig. 4) with $\beta=0.1$ is considered for all the converters where stations \#1 and \#4 (\#2 and \#3) export (import) about $900 \mathrm{MW}$ each. As expected, the remaining negative pole converters share the loss of $450 \mathrm{MW}$ and the resultant unbalance in the metallic return currents in equal proportion while maintaining the positive pole power (Fig. 9). The loss of one rectifier has resulted in an increase in power share by the other rectifying unit (in station \#2) from -450 MW to $-600 \mathrm{MW}$ and a similar decrease for both the inverter units (in station \#1 and \#3).

It can be seen that there is a very close match between these results except that the switching ripple is absent in the averaged model. Moreover, the converter outage is slower in the EMTDC/PSCAD model since the ac breaker opening considers natural current zero in each phase while the averaged model considers almost instantaneous switching. This validates the modal analysis with the averaged model reported in the previous section. The dynamic response from the detailed switched model is reported throughout the rest of the paper.

\section{B. Problems with Droop Control using Local Voltage}

As mentioned in Section III-A due to non-unique power flow solution with the local voltage based droop control, a 
common DC bus voltage reference signal needs to be communicated to all the converter locations. We have done several tests to validate this problem and one of them is presented here to establish the point. As shown in Fig. 10 the negative pole converters share the power change equally for equal droop

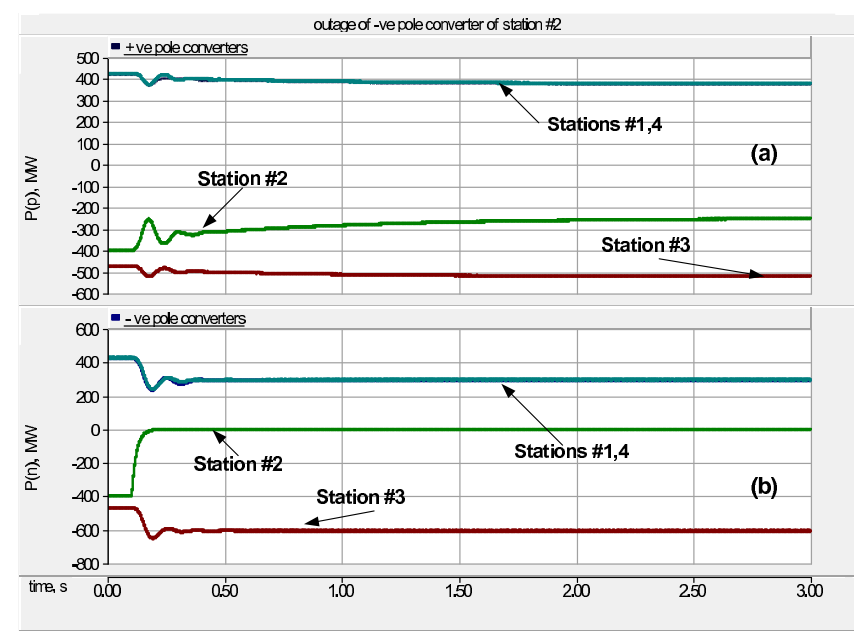

Fig. 10. Local signal based autonomous power sharing following the outage of the negative pole converter at converter station \#2.

coefficients following the negative pole converter outage at station \#2. However, the power in positive pole converters drift unacceptably to reach a different post-contingency operating point from their nominal values. On the contrary, common reference signal based droop control does not suffer from this issue and thus it is justifiable to use this architecture also for the proposed adaptive (variable) droop scheme.

\section{Fixed vs Adaptive (variable) Droop}

In this section the performance of the fixed and adaptive (variable) droop scheme is compared in the backdrop of no power sharing control (no droop) wherein only converter station \#1 is under DC link voltage control (slack). For the fixed droop, $\beta=1 \%$ was chosen while $\lambda=2$ was used for variable droop. Unequal loading conditions at the four converter stations were considered to demonstrate the benefit of adaptive droop. Both rectifier and inverter outages were simulated under a number of scenarios out of which two representative cases are presented below.

1) Inverter Outage: In the first case each converter pole in stations \#1 and \#4 exports about $420 \mathrm{MW}$ and $200 \mathrm{MW}$, respectively from the DC grid, while each of those in stations \#2 and \#3 import around $310 \mathrm{MW}$ into it. The outage of the negative pole converter in the inverter station \#4 was considered. It can be seen from Fig. 11(a) that in absence of droop control the slack converter station (\#1) operating in $V_{D}-Q$ mode increases its power to $600 \mathrm{MW}$ to compensate the loss of the inverter.

This results in the overloading of the unit whereas the inverter in stations \#2 (Fig. 11(b)) and \#3 (not shown) maintains the pre-contingency power.

With fixed droop the burden is shared equally by the remaining converters in the negative pole as shown in Fig. 12.

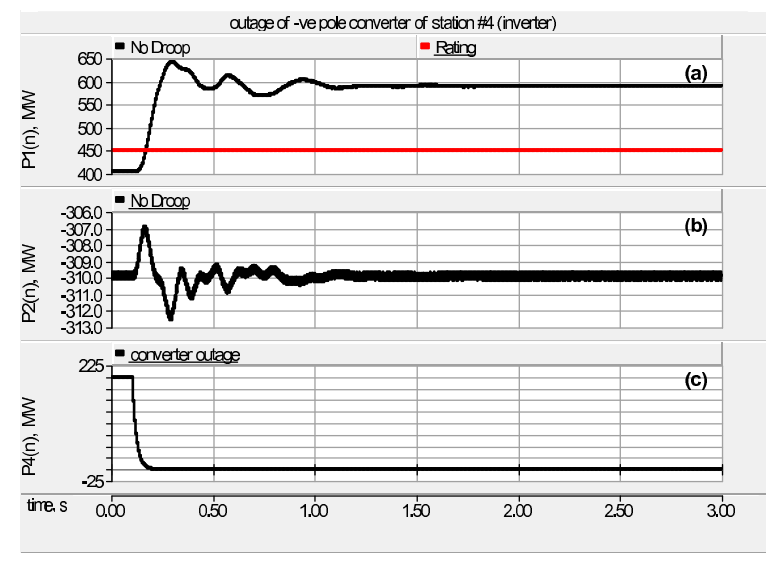

Fig. 11. Power sharing without droop scheme following the outage of the negative pole converter (inverter) of the converter station \#4.

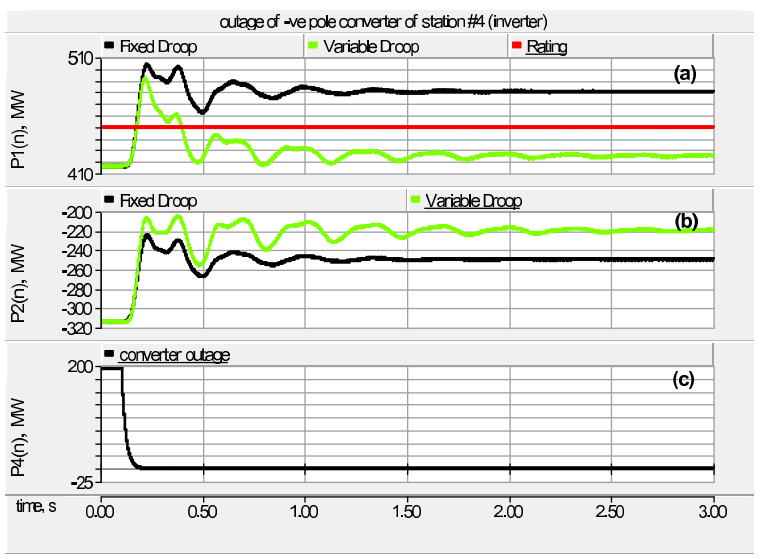

Fig. 12. Comparison of power sharing for fixed vs variable droop scheme following the outage of the negative pole converter (inverter) of the converter station \#4.

However, this does not account for the available converter headroom - which in this particular case is only $30 \mathrm{MW}$ for each converter in station \#1. Therefore, the inverting unit at that station shares about $66 \mathrm{MW}$ power and violates the power rating of $450 \mathrm{MW}$ as shown in Fig. 12(a). Adaptive droop control ensures a higher droop value for lower headroom and prevents station \#1 from overloading, see Fig. 12(a).

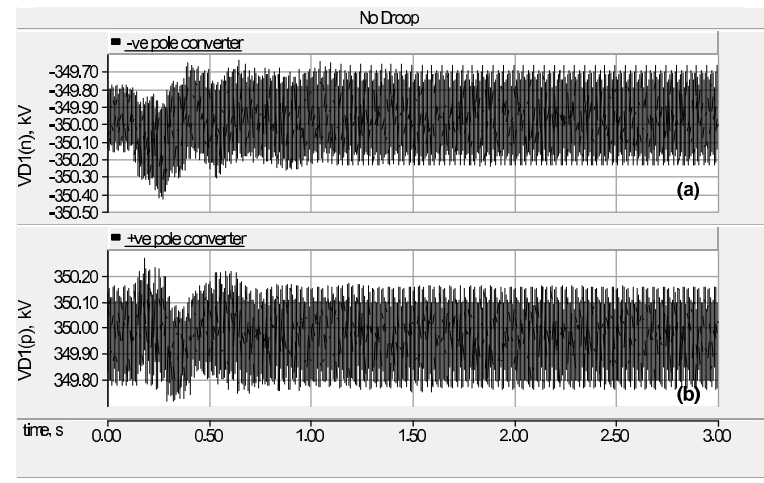

Fig. 13. DC link voltage of the converter in $V_{D}-Q$ control mode following the outage of the negative pole converter (inverter) of the converter station \#4. 
Variation of the DC link voltage at converter station \#1 is shown in Figs 13 and 14 for no droop, variable and fixed droops, respectively.

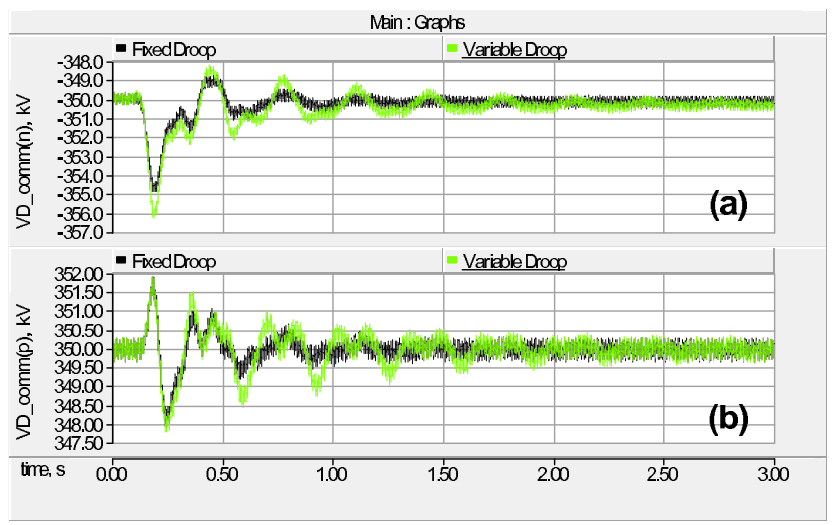

Fig. 14. Comparison of common bus DC link voltage for fixed vs variable droop scheme following the outage of the negative pole converter (inverter) of the converter station \#4.

The DC bus voltage of the slack converter (no droop) is maintained constant at $\pm 350 \mathrm{kV}$ with very little overshoot in its transient response. This is larger for the common bus voltage for fixed and variable droop control. It can be seen from Fig. 14 that the proposed adaptive droop scheme does not deteriorate the DC link dynamics further than the fixed droop scheme.

2) Rectifier Outage: In this case, converter pole in stations \#2 and \#3 act as rectifiers importing about $200 \mathrm{MW}$ and 420 MW respectively into the MTDC grid, while those in stations \#1 and \#4 work as inverters carrying around 310 MW each. The outage of the negative pole converter in the rectifying station \#2 was considered.

Dynamic response of the system with no droop following the contingency are shown in Fig. 15.

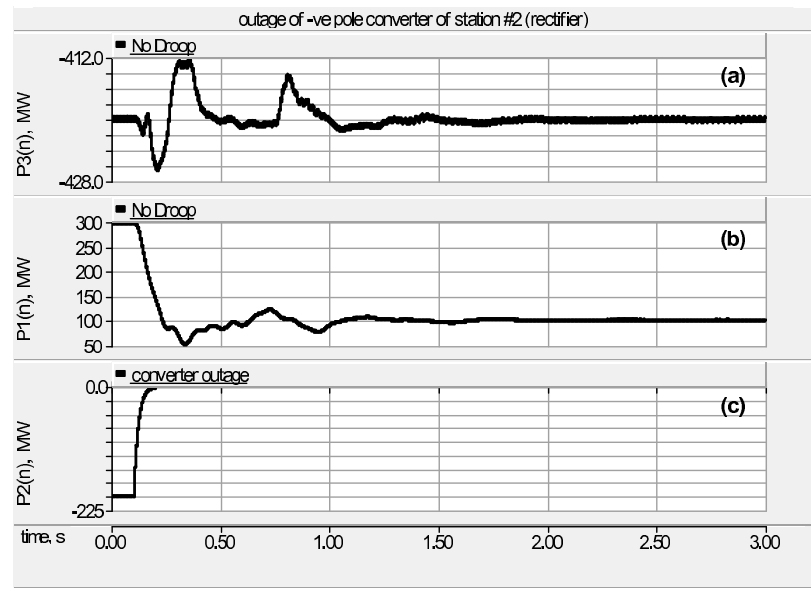

Fig. 15. Power sharing without droop scheme following the outage of the negative pole converter (rectifier) of the converter station $\# 2$.

Although no converter has violated their power ratings, the large drop in station \#1 power by about 200 MW might not be acceptable for the ac side utility connected to this station. It can be seen from Fig. 16 that the proposed scheme is effective for the rectifier outage scenario too.



Fig. 16. Comparison of power sharing for fixed vs variable droop scheme following the outage of the negative pole converter (rectifier) of the converter station \#2.

It is to be noted that the post-outage steady-state operating points in above simulation results agree with the analytical values shown in Table II which were obtained from the formulation in Section IV.

TABLE II

POST CONTINGENCY STEADY-STATE OPERATING POINTS WITH FIXED AND VARIABLE DROOP CONTROL FOR RECTIFIER AND INVERTER OUTAGES

\begin{tabular}{|c||c|c|c|c||c|c|c|c|}
\hline \multicolumn{1}{|c||}{} & \multicolumn{4}{|c||}{ Inverter outage } & \multicolumn{4}{c|}{ Rectifier outage } \\
\cline { 2 - 9 } & pre & $H_{j}$ & fixed & variable & pre & $H_{j}$ & fixed & variable \\
\hline \hline P1 & 417.2 & 32.8 & $\mathbf{4 8 3 . 9}$ & 422.8 & 307.6 & 142.4 & 241.0 & 209.5 \\
\hline P2 & -312.8 & 137.2 & -246.1 & -215.6 & -202.4 & 247.6 & N/A & N/A \\
\hline P3 & -312.8 & 137.2 & -246.1 & -215.6 & -422.4 & 27.6 & $\mathbf{- 4 8 9 . 0}$ & -426.1 \\
\hline P4 & 197.2 & 252.8 & N/A & N/A & 307.6 & 142.4 & 241.0 & 209.5 \\
\hline
\end{tabular}

Note that in practice; there will be limits on $i_{d j}^{\prime *}$ and $i_{q j}^{*}$ of the current controller. In the case studies shown above, these limits on $i_{d j}^{\prime *}$ and $i_{q j}^{\prime *}$ were ignored for illustrative purposes to validate the post contingency operating condition obtained from the analytical expression through time domain non-linear simulation as shown in Table II.

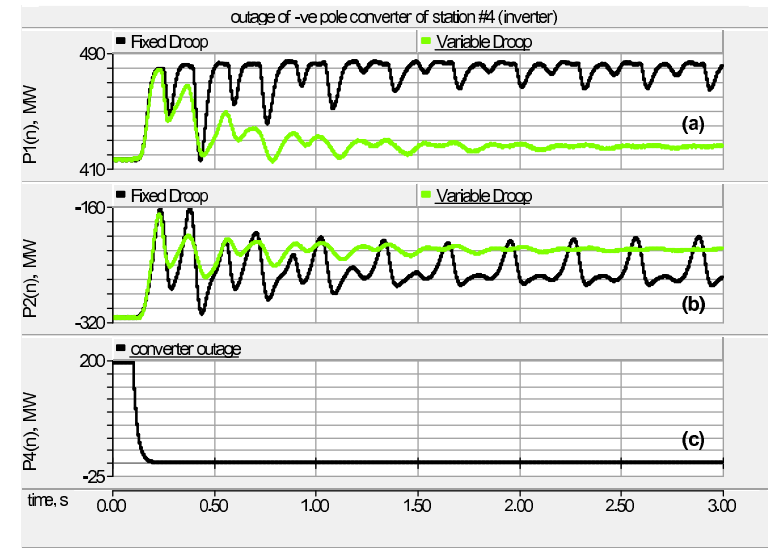

Fig. 17. Comparison of power sharing for fixed vs variable droop scheme following the outage of the negative pole converter (inverter) of the converter station \#4. Converter current control limits are in place.

The simulation results with the current controller limits in place are shown in Figs 17, 18 for inverter outage condition. 
The current limiting strategy is based on limiting the magnitude of the current vector in $d-q$ frame and re-computing the values of $i_{d j}^{\prime *}$ and $i_{q j}^{\prime *}$ while keeping the phase of the vector intact.

Fig. 17 shows the response of the converter real powers following the outage of the negative pole of converter station \#4 under the operating condition described in Section VII-C1. Due to repeated hitting of current controller limits with fixed droop control, the power sharing amongst the converter stations are jeopardized and the dynamic behavior is poorer (see, Fig. 17(a) and (b)). On the other hand, the variable droop scheme leads to desirable system performance.

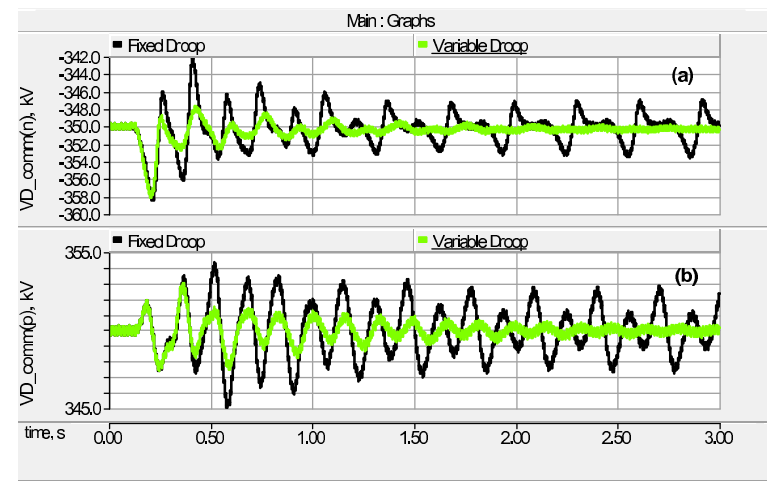

Fig. 18. Comparison of common bus DC link voltage for fixed vs variable droop scheme following the outage of the negative pole converter (inverter) of the converter station \#4. Converter current control limits are in place.

As shown in Fig. 18, there is larger variation in DC link voltage when a fixed droop control is employed while the proposed strategy results in much tighter DC link voltage control.

\section{CONCLUSION}

Limitations of using a fixed active power-DC link voltage droop for autonomous power sharing in MTDC grids is demonstrated. A novel scheme for adapting the droop coefficients to share the burden according to the available headroom of each converter station is proposed. Advantage of this adaptive droop scheme for autonomous power sharing is established through transient simulations on an MTDC grid with four bipolar converter stations and DC cable network with metallic return. Results for both rectifier and inverter outages under two different scenarios are presented. Post contingency steady-state operating points obtained from transient simulation are shown to be consistent with those derived analytically. Impact of varying droop coefficients on the stability of the MTDC grid is established.

\section{REFERENCES}

[1] J. Blau, "Europe plans a north sea grid," IEEE Spectrum, vol. 47, no. 3, pp. 12-13, 2010.

[2] K. Rudion, A. Orths, P. B. Eriksen, and Z. A. Styczynski, "Toward a benchmark test system for the offshore grid in the north sea," in IEEE Power and Energy Society General Meeting, 2010, 2010, pp. 1-8.

[3] X. Lie, B. W. Williams, and Y. Liangzhong, "Multi-terminal dc transmission systems for connecting large offshore wind farms," in IEEE Power and Energy Society General Meeting - Conversion and Delivery of Electrical Energy in the 21st Century, 2008, 2008, pp. 1-7.
[4] Z. Shu, L. Jun, J. B. Ekanayake, and N. Jenkins, "Control of multiterminal vsc-hvdc transmission system for offshore wind power generation," in Proceedings of the 44th International Universities Power Engineering Conference (UPEC), 2009, 2009, pp. 1-5.

[5] B. Berggren, R. Majumder, C. Sao, and K. Linden, "Method and control device for controlling power flow within a dc power transmission network," WIPO International Publication Number WO 2012/000549 A1, international filing date: 30 June 2010; international publication date: 5 January 2012.

[6] T. M. Haileselassie and K. Uhlen, "Primary frequency control of remote grids connected by multi-terminal HVDC," in IEEE Power and Energy Society General Meeting, 2010.

[7] R. Majumder, B. Chaudhuri, A. Ghosh, G. Ledwich, and F. Zare, "Improvement of stability and load sharing in an autonomous microgrid using supplementary droop control loop," IEEE Transactions on Power Systems, vol. 25, no. 2, pp. 796-808, 2010.

[8] C. Schauder and H. Mehta, "Vector analysis and control of advanced static var compensators," IEE Proceedings on Generation, Transmission and Distribution, vol. 140, no. 4, pp. 299-306, 1993.

[9] S. R. Sanders, J. M. Noworolski, X. Z. Liu, and G. C. Verghese, "Generalized averaging method for power conversion circuits," IEEE Transactions on Power Electronics, vol. 6, no. 2, pp. 251-259, 1991.

[10] N. Mohan, T. M. Undeland, and W. P. Robbins, Power electronics : converters, applications, and design, 3rd ed. Hoboken, N.J. ; [Chichester]: Wiley, 2003.

[11] N. R. Chaudhuri, R. Majumder, B. Chaudhuri, and J. Pan, "Stability analysis of VSC MTDC grids connected to multi-machine AC systems," accepted for publication in IEEE Transactions on Power Delivery.

[12] N. R. Chaudhuri, R. Majumder, B. Chaudhuri, J. Pan, and R. Nuqui, "Modeling and stability analysis of MTDC grids for offshore wind farms: A case study on the north sea benchmark system," to appear in Proceedings of IEEE PES General Meeting, 2011.

[13] Z. Lidong, L. Harnefors, and H. P. Nee, "Power-synchronization control of grid-connected voltage-source converters," IEEE Transactions on Power Systems, vol. 25, no. 2, pp. 809-820, 2010

[14] J. Arrillaga and B. D. Smith, AC-DC power system analysis. Stevenage: Institution of Electrical Engineers, 1998.

[15] P. Kundur, Power system stability and control, ser. The EPRI power system engineering series. New York; London: McGraw-Hill, 1994.

Nilanjan Ray Chaudhuri (S'08-M'09) received his $\mathrm{PhD}$ in electrical power systems from Imperial College London in 2011. He was with GE, John F. Welch Technology Center, Bangalore, India, till 2007. He was a research scholar at the University of Western Ontario (UWO), London, ON, Canada. His research interests include power system dynamics and control, application of power electronics in power systems, online system identification, FACTS, HVDC and renewable energy systems.

Balarko Chaudhuri (S'02-M'06-SM'11) received his $\mathrm{PhD}$ in electrical power systems from Imperial College London in 2005. He worked with General Electric Global Research for a year before coming back to Imperial College as a post-doctoral research associate in 2006. He is presently a senior lecturer in the department of Electrical and Electronic Engineering at Imperial College London. His research interests are in the areas of power system dynamics and stability, FACTS/HVDC, robust control and renewable energy. 